\title{
Features of Structural Relaxation in Diblock Copolymers
}

\author{
Mingchao Ma, ${ }^{1,2}$ Tianju Xue, ${ }^{1,2}$ Shenyue Chen, ${ }^{1,2}$ Yunlong Guo, ${ }^{, 1,2,3}$ Yujie Chen,,3 and Hezhou Liu ${ }^{2,3}$ \\ ${ }^{1}$ The State Key Lab of Metal Matrix Composites, ${ }^{2}$ University of Michigan - Shanghai Jiao Tong University Joint Institute, \\ and ${ }^{3}$ School of Materials Science and Engineering, Shanghai Jiao Tong University, Shanghai 200240, China
}

\begin{abstract}
Time- and temperature-dependent structural relaxation (physical aging) of poly(styrene- $b$-methyl methacrylate) (PS- $b$-PMMA) block copolymers was investigated by calorimetry. Our study reveals the interplay of the relaxation responses of the two components of the copolymer in an intermediate temperature regime. That is, when the testing temperature is closely below the glass transition temperatures of PS and PMMA, structural relaxation in these polymer phases takes place concurrently, the corresponding thermogram displays partially superposed dual endothermic peaks as a feature of physical aging in the diblock copolymers. The aging response for each component is identified from a curve fitting method and analyzed by the relaxation of enthalpy. Comparing with the homopolymer analogs, the PS and PMMA in diblock copolymers show enhanced aging rate.
\end{abstract}

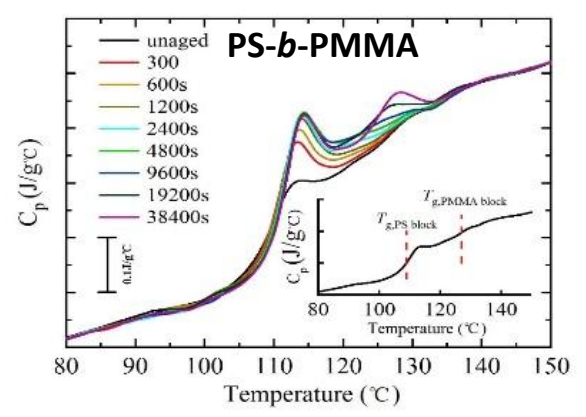

KEYWORDS: structural relaxation, diblock copolymers, enthalpy recovery, calorimetry

\section{Introduction}

Block copolymers usually serve as templates and scaffolds in fabrication of patterned or hierarchical structures down to nanometer scale, so that design and self-assembly of structures from copolymers by phase separation have received vast research interests in last two decades.[1-6] Many procedures to create complicated architectures ranging from uniform ordered planar nanostructures[7] to three-dimensional hierarchical structures[8] have been developed. While the smart design and processing utilizing copolymers have attracted a great deal of attention, the naturally time-dependent instability of the material, including physical aging which affects the physical properties of the chemically immiscible blocks, especially how the properties evolve in the relaxation process, and how they interact each other during aging, remain to be elucidated.

Below the glass transition temperature $\left(T_{\mathrm{g}}\right)$, polymeric materials often fall into non-equilibrium state, the conformation of disordered molecules alter with time, toward a lower position in the potential energy landscape. This evolution process is referred to as structural relaxation, or physical aging.[9, 10] Many physical properties of polymers such as specific volume, mechanical moduli, gas permeability, and thermal conductivity change concurrently in the process of physical aging.

Physical aging is of highly technological importance, however, to the best of our knowledge, structural relaxation of copolymers has not been systematically investigated, although a lot of research effort exists for homopolymers.[9, 11-13] To date the majority of literature related to physical aging of copolymers focuses on particular engineering properties of the material, for instance, a few studies reported the influence of processing on mechanical behavior[14-16] or free volume change[17] in copolymers during aging, and several others examined the physical aging of blends of homopolymer and copolymer, regarding dynamic mechanical response or me- chanical strength and elasticity[18, 19]. Lack of fundamental understanding and systematic characterization of physical aging of copolymers would bottleneck applications of the material in the aforesaid emerging fields.

Experimentally, due to its high sensitivity and low amount need of materials, thermal analysis is a routine means to characterize aging response from enthalpy recovery, and it has been widely applied to capture aging effect of homopolymers.[13] In contrast, for copolymers, this method has not been adequately exploited to describe structural relaxation of the materials.[20]

We systematically investigated the structural relaxation of copolymers using a differential scanning calorimeter (DSC). Here we report the features of physical aging of a representative diblock copolymer, i.e., poly(styrene- $b$-methyl methacrylate) (PS- $b$-PMMA), which serves as a model system in many studies of block copolymers. The aging features are unique not only in that the aging response of the two blocks deviate from bulk due to nanoscopic confinement induced by phase separation,[21] but also because the aging response of the two components were found interacting with each other as PS and PMMA have close $T_{\mathrm{g}} \mathrm{s}$.

\section{Experimental}

\subsection{Materials}

The atactic polystyrene-poly(methyl methacrylate) diblock copolymer was purchased from Polymer Source Inc. and used as received. The material has weight-average molecular weight of PS component $\left(\mathrm{M}_{\mathrm{w}, \mathrm{PS}}\right)$ of $259,000 \mathrm{~g} / \mathrm{mol}$ and weight-average molecular weight of PMMA component $\left(\mathrm{M}_{\mathrm{w}, \mathrm{PMMA}}\right)$ of $629,000 \mathrm{~g} / \mathrm{mol}$. The component percentage of the material is $29 \% / 71 \%$. In copolymer synthesis process, the molecular weight and polydispersity index (PDI) of polystyrene block was measured by size exclusion chromatography 
before addition of MMA monomer. The final block copolymer composition was calculated from 1H-NMR spectroscopy and PDI of 1.08 was determined by SEC.

The homopolymers PS and PMMA used in control experiments were purchased from Sigma-Aldrich and American Polymer Standards Corporation respectively and used as received. The $M_{\mathrm{W}}$ of homo-PS is $254 \mathrm{~kg} / \mathrm{mol}$ and PDI is 2.83 , and the $M_{\mathrm{W}}$ of homo-PMMA is $620 \mathrm{~kg} / \mathrm{mol}$ and PDI is 1.05 . For better comparison, both of the homopolymers have the same molecular weight with the corresponding polymer blocks in the copolymer sample.

\subsection{Testing Method}

Physical aging measurements were carried out by a Shimadzu DSC-60 plus DSC with nitrogen gas environment. The instrument was calibrated by standard Indium. In aging testing, a 3 5 mg sample was first sealed in an aluminum pan and it was subsequently heated to $180^{\circ} \mathrm{C}$ (well above the glass transition temperature of the both polymer components) with a constant ramp rate of $20^{\circ} \mathrm{C} / \mathrm{min}$ and held for $10 \mathrm{~min}$ to remove the residual stress. Then the sample was quenched to a desired aging temperature with constant cooling rate of $-30^{\circ} \mathrm{C} / \mathrm{min}$ and annealed for an aging time $\left(t_{a}\right)$. After that, the sample was quenched to $40^{\circ} \mathrm{C}$ (well below the glass transition temperature of PS and PMMA components) with same cooling rate -30 ${ }^{\circ} \mathrm{C} / \mathrm{min}$ and then reheated to $180^{\circ} \mathrm{C}$ at heating rate of $20^{\circ} \mathrm{C} / \mathrm{min}$. The thermogram of the second heating scan was recorded to determine the aging response of the material. The aging testing protocol is represented by Figure 1.

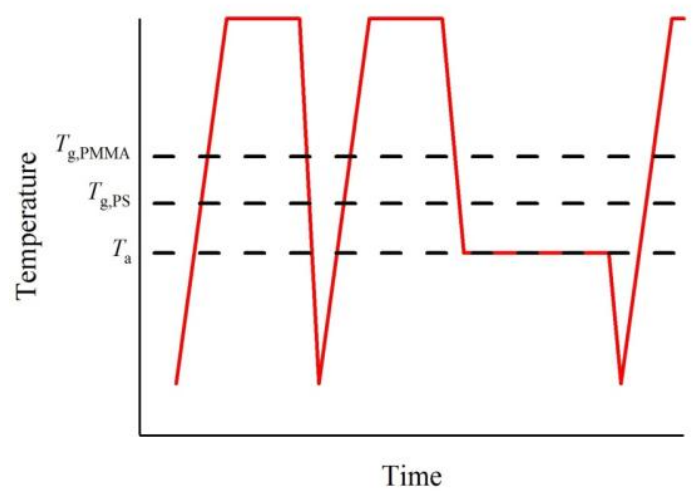

Figure 1. Temperature protocol of aging experiments

\section{Results and Discussion}

Figure 2 depicts representative results of structural relaxation for a PS- $b$-PMMA sample (mole fraction of PS block $f_{\mathrm{PS}}=$ $0.29, T_{\mathrm{g}, \text { Ps-block }}=109{ }^{\circ} \mathrm{C}$, and $T_{\mathrm{g} \text {, PMMA-block }}=127{ }^{\circ} \mathrm{C}$, determined from the second heating of the DSC trace) at three aging temperatures. As shown in Fig. 2(a), at $89{ }^{\circ} \mathrm{C}$, i.e., $20{ }^{\circ} \mathrm{C}$ lower than the $T_{\mathrm{g} \text {, Ps-block }}$, enthalpy recovery peaks of the PS block were observed growing with increasing aging times, while the aging response of PMMA block was depressed in our experimental timescale due to a large departure of $T_{\mathrm{g}}$, PMMA-block $T_{\text {aging }}=38{ }^{\circ} \mathrm{C}$. In contrast, when $T_{\text {aging }}=114{ }^{\circ} \mathrm{C}$, aging response of PMMA block was clearly captured by the heat capacity curves in Fig. 2(b). As $T_{\text {aging }}>T_{\text {g, PS-block, the PS block }}$ was immediately set at equilibrium in its liquid state after quenching to $T_{\text {aging. }}$ Figure 2(c) illustrates the coupled aging response of PS and PMMA components in the thermogram when $T_{\text {aging }}=99{ }^{\circ} \mathrm{C}$. Other than this particular representative aging temperature, actually in an intermediate temperature regime closely below $T_{\mathrm{g}}$, Ps-block, the structural relaxation response contributed from both PS and PMMA blocks was observed. Owing to the close $T_{\mathrm{g}} \mathrm{s}$ of the two components, the overall response of physical aging demonstrated an overlay of DSC traces that correspond to the enthalpy recovery of PS and PMMA blocks, respectively. In our aging times up to 38400 seconds, except that the PS block approached equilibrium, as shown in Fig.2(c), aging processes of PMMA block kept ongoing during the entire timescale of the testing. 

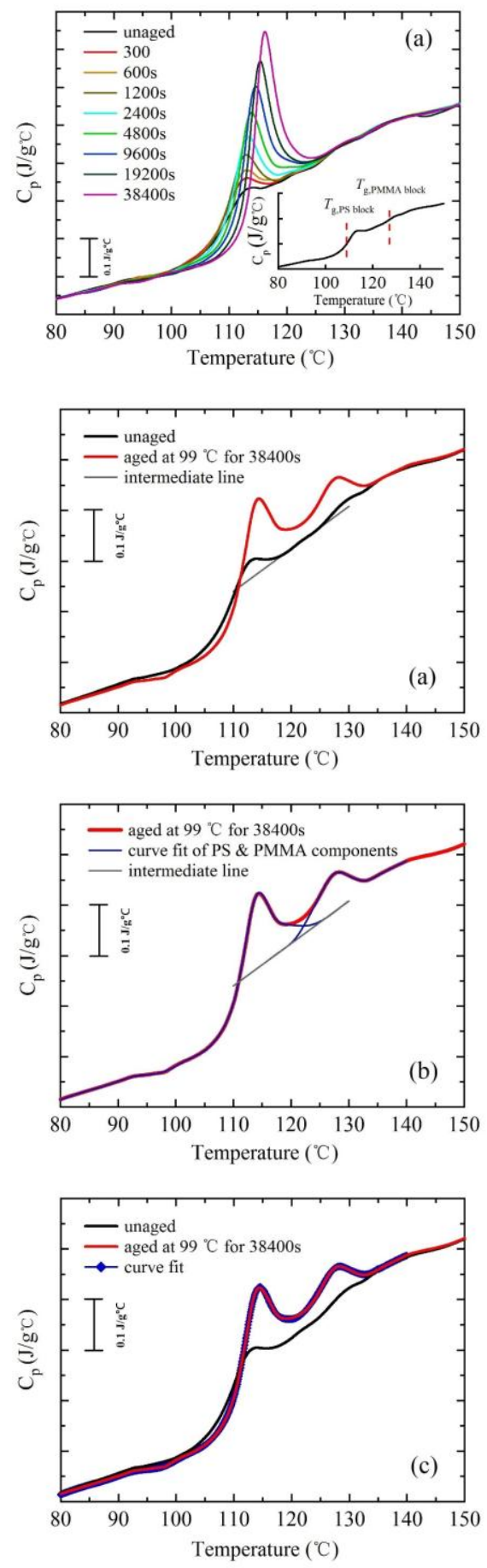

Figure 3. Curve fit of overlapped aging response between PS and PMMA blocks. (a) Determination of a baseline indicating an intermediate state. (b) Gaussian fit of individual aging response. (c) Sum of curve fits recovers the raw aging data.

To identify aging response of each component individually, we utilized a curve fitting method on the data sets when the overlapping aging results occurred. Figure 3(a) shows the aging response when the sample was aged at $99{ }^{\circ} \mathrm{C}$ for 38400 seconds. As a reference, the unaged curve provides heat capacity data of the PS and PMMA through glass transitions. The grey line is the linear portion of the intermediate state at which the PS phase has transferred into liquid while the
PMMA phase remains in glassy state. We hypothesize that the overall aging response in the DSC trace between the $T_{\mathrm{g}} \mathrm{S}$ comes from the superposition of the aging response of each component. The curve fitting of the component aging starts from (PMMA phase) or ends upon the grey reference line (PS phase), as shown in Fig. 3(b), according to the thermogram of the baseline. In this figure, the individual heat capacity curve in blue, obtained from Gaussian fit, represents the aging response of PS or PMMA in the copolymer. The summation of these curve fitting results can recover the raw data, as illustrated in Fig. 3(c). As such, the curve fitting separates overlapped aging response in the diblock copolymer, the resultant family of aging curves for each component can be analyzed by the approach established for enthalpy recovery in homopolymers. The aging curves for each component were obtained from curve fitting based on the DSC trace and intermediate state, the accuracy and robustness of the obtained aging response was tested and approved from aging of a homopolymer. That is, we use a part of the aging data $(50 \%)$ and the baseline to generate the curve fit of aging response, and compare the curve fit with the whole data set and found they match each other very well. The results indicate that given partial availability of the calorimetric peak and baseline in the differential thermogram, the entire aging response could be identified from curve fitting in a relatively narrow temperature regime.
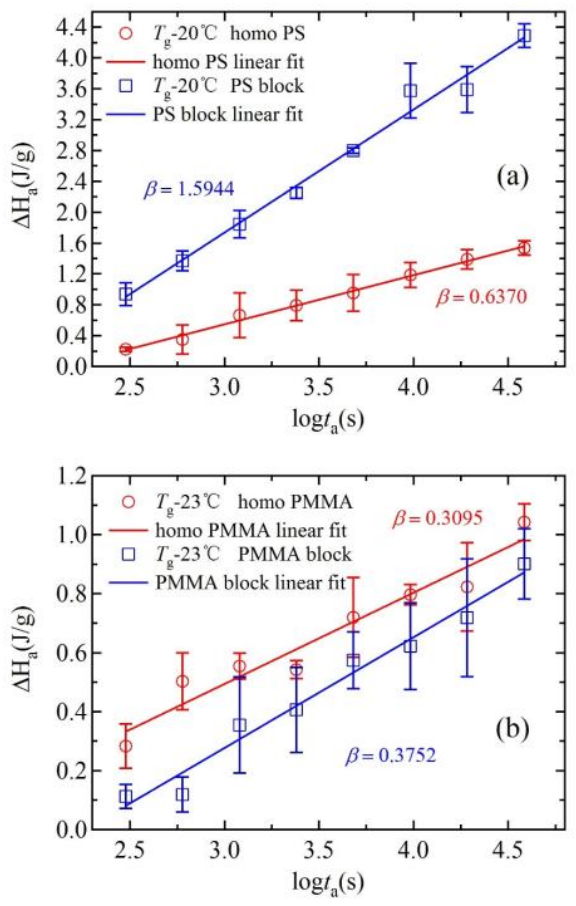

Figure 4. Relaxed enthalpy of PS and PMMA in diblock copolymers and in homopolymers: (a) PS, $T_{\mathrm{g}}-T_{\text {aging }}=20^{\circ} \mathrm{C}$ and (b) PMMA, $T_{\mathrm{g}}-T_{\text {aging }}=23^{\circ} \mathrm{C}$.

The amount of enthalpy relaxed during aging was evaluated by the discrepancy between integration of thermograms of aged and unaged samples, as expressed by the following equation:

$$
\Delta H_{a}=\int_{T_{1}}^{T_{2}}\left(C_{p, \text { aged }}(T)-C_{p, \text { unaged }}(T)\right) d T
$$


where $C_{\mathrm{p}, \text { aged }}$ and $C_{\mathrm{p} \text {,unaged }}$ are the heat capacity data determined from DSC traces of aged and unaged samples, and $T_{1}$ and $T_{2}$ are reference temperatures in glassy and liquid state, respectively, at which the baselines of aged and unaged samples coincide.

Figure 4 shows relaxed enthalpy of PS and PMMA phases in the copolymer, compared with their homopolymer counterparts with similar molecular weights. When $T_{\mathrm{g}}-T_{\text {aging }}=20^{\circ} \mathrm{C}$ or $23{ }^{\circ} \mathrm{C}$, for PS and PMMA respectively, the aging process of both homopolymer and copolymer blocks remains ongoing within 38400 seconds. In logarithmic time scale, $\Delta H_{\mathrm{a}}$ increases with aging time in a closely linear manner for all cases. This trend suggests that the data captured an intermediated stage of enthalpy recovery, as the value of time rate of $\Delta H_{\mathrm{a}}$ is much lower and alters with time when aging process starts at $T_{\text {aging, }}$, or, isothermally approaches to the equilibrium plateau. The aging rate $\beta$, determined by the slope of the linear fit, measures the speed of enthalpy change to a lower position in potential energy landscape, toward equilibrium. As shown in Fig. 4, the $\beta$ values of copolymer blocks were found greater than those in homopolymers, when the departure of $T_{\text {aging }}$ from $T_{\mathrm{g}}$ was kept same in all aging scenarios. According to the temperature protocol of our aging testing, prior to isothermal aging, the sample was first heated up to and annealed at 180 ${ }^{\circ} \mathrm{C}$ for 10 minutes. This thermal treatment should induce phase separation of PS and PMMA blocks,[21] and the micro domains was almost retained during quenching and the subsequent structural relaxation. As such, component blocks in the copolymer confine each other due to phase separation. The size effect and confinement effect may account for the differences in enthalpy recovery from the bulk materials, although their molecular weights equal.

Next we compare our results with the findings on physical aging of polymer nanocomposites, in which the polymer matrix is considered under three dimensional confinement as well. Note that the accelerated or depressed aging process in polymer nanocomposites often accompanies with $T_{\mathrm{g}}$ deviation, but there is no unanimous consensus detected on how $T_{\mathrm{g}}$ varies from that of the bulk, i.e., the $T_{\mathrm{g}}$ could be either increased or decreased, even no change when compared with the $T_{\text {g,bulk. }}$ [22, 23] The equilibrium recovery rate of polymer nanocomposites is found reduced from that of the bulk, when the $T_{\mathrm{g}}$ increases. In our study, the $T_{\mathrm{g}}$ of polymer block departs $+3{ }^{\circ} \mathrm{C}$ or $-3{ }^{\circ} \mathrm{C}$ from the bulk $T_{\mathrm{g}}$, for PS and PMMA, respectively. In nanocomposite studies, a mutual finding is that the magnitude of $T_{\mathrm{g}}$ deviation (usually $<10 \mathrm{~K}$ ) is milder than that in thin films. Although the magnitude of $T_{\mathrm{g}}$ deviation observed in current investigation is comparable to the results in literature of polymer nanocomposites, the trend of $T_{\mathrm{g}}$ changes here is not in line with the those in PS (decreased) or PMMA (increased or no change) nanocomposites.[22] Notwithstanding this discrepancy might be ascribed to softer confinement condition surrounding each microphase, consider the small magnitude of the $T_{\mathrm{g}}$ deviation, how the $T_{\mathrm{g}}$ of copolymer component changes with chemical structure, molecular weight, component fraction, etc. needs to understood by a further study focusing on it. In current work, both the PS and PMMA phases in diblock copolymer showed enhanced enthalpy relaxation rate, this result is consistent with the results of PS/silica,[24] PMMA/silica,[25] and PS/gold nanocomposites,[26] in which the properties of polymeric materials were found evolving toward faster equilibrium achievement during physical aging.

Differing from nanocomposites, in this study, each component in block copolymers is confined by its chemically immiscible partner, instead of rigid nanofillers. This confinement environment, together with the chemical bond connection between the blocks, might be able to induce some intriguing features of segmental dynamics and structural relaxation, hence the features could be influenced by the mole fraction, molecular weight, chemical structure, etc.

To examine the temperature effect of physical aging, the aging time was fixed at 19200 seconds, resultant aging response was recorded at various temperatures, as illustrated in Figure 5. As $T_{\text {aging }}$ increases, the calorimetric peak gradually transits from PS- to PMMA-corresponded temperature range. The peak of PS thermogram decreases with increasing $T_{\mathrm{a}}$ from 89 to $104{ }^{\circ} \mathrm{C}$ because the limit of enthalpy span in aging is reduced. In contrast, the calorimetric peak of PMMA block is enhanced when $T_{\text {aging }}$ increases, in accordance with elevated driving force of aging when the material getting closer to $T_{\mathrm{g}}$.

In summary, structural relaxation of an amorphous diblock copolymer PS- $b$-PMMA was measured by DSC. Due to the close $T_{\mathrm{g}} \mathrm{s}$ of the two components, the aging response overlapped in an intermediate temperature regime. The coupled aging response can be separated by curve fitting. With regard to diblock copolymers, the aging rate was greater than that in the bulk materials. The confinement induced from phase separation which was set up before aging may be responsible for the enhanced relaxation and the $T_{\mathrm{g}}$ deviation. The results suggest that there exists unique glassy behavior associated with structural relaxation in block copolymers, in that the copolymer material provides considerable room to alter the geometry and intensity of nanoconfinement, as well as the composition and molecular weight.

\section{AUTHOR INFORMATION}

\section{Corresponding Author}

*E-mail: yunlong.guo@sjtu.edu.cn

\section{Notes}

The authors declare no competing financial interests.

\section{ACKNOWLEDGMENT}

The authors acknowledge the start-up fund of Y.G. from both University of Michigan - Shanghai Jiao Tong University Joint Institute, and School of Materials Science and Engineering at SJTU. The authors also acknowledge the National Science Foundation of China for financial support through the General Program 2157408, and the foundation of Shanghai Sailing Plan 16YF1406100. Y.G. is very grateful to the National Youth 1000 Talent Program of China, the Shanghai 1000 Talent Plan, and the support by the Scientific Research Foundation for the Returned Overseas Chinese Scholars, State Education Ministry of China.

\section{REFERENCES}


[1] F.S. Bates, G.H. Fredrickson, Block CopolymersDesigner Soft Materials, Physics Today, 52 (1999) 32-38.

[2] Z. Chen, J.A. Kornfield, S.D. Smith, J.T. Grothaus, M.M. Satkowski, Pathways to Macroscale Order in Nanostructured Block Copolymers, Science, 277 (1997) 1248-1253.

[3] J.Y. Cheng, A.M. Mayes, C.A. Ross, Nanostructure engineering by templated self-assembly of block copolymers, Nature Materials 3(2004) 823-828.

[4] S.B. Darling, Directing the self-assembly of block copolymers, Progress in Polymer Science, 32 (2007) 11521204.

[5] S. Jain, F.S. Bates, On the Origins of Morphological Complexity in Block Copolymer Surfactants, Science, 300 (2003) 460-464.

[6] A. Ruzette, L. Leibler, Block copolymers in tomorrow's plastics, Nature Materials, 4 (2005) 19-31.

[7] S. Park, D.H. Lee, J. Xu, B. Kim, S.W. Hong, U. Jeong, T. Xu, T.P. Russell, Macroscopic 10-Terabit-per-SquareInch Arrays from Block Copolymers with Lateral Order, Science, 323 (2009) 1030-1033.

[8] H. Sai, K.W. Tan, K. Hur, E. Asenath-smith, R. Hovden, J. Y., M. Riccio, D.A. Muller, V. Elser, L.A. Estroff, S.M. Gruner, U. Wiesner, Hierarchical Porous Polymer Scaffolds from Block Copolymers, Science, 341 (2013) 530-534.

[9] I.M. Hodge, Physical Aging in Polymer Glasses, Science, 267 (1995) 1945-1947.

[10] L.C.E. Struik, Physical Aging in Amorphous Polymers and Other Materials, Elsevier Scientific Publishing Co., Amsterdam, 1978.

[11] Y. Guo, R.D. Bradshaw, Isothermal Physical Aging Characterization of Polyether-ether-ketone (PEEK) and Polyphenylene Sulfide (PPS) Films by Creep and Stress Relaxation, Mechanics of Time-Dependent Materials, 11 (2007) 61-89.

[12] Y. Guo, R.D. Bradshaw, Long-term creep of polyphenylene sulfide (PPS) subjected to complex thermal histories: The effects of nonisothermal physical aging, Polymer, 50 (2009) 4048-4055.

[13] C.T. Moynihan, P.B. Macedo, C.J. Montrose, P.K. Gupta, M.A. Debolt, J.F. Dill, B.E. Dom, P.W. Drake, A.J.

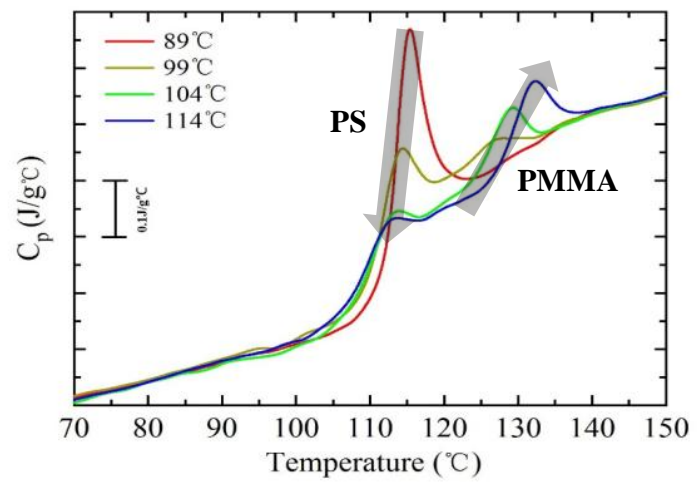

Figure 5. Physical aging response of PS- $b$-PMMA at various aging temperatures, while the aging time was kept as 19200 seconds. The arrows indicate the trend of enthalpy relaxation peak changing with increasing aging temperature.
Easteal, P.B. Elterman, R.P. Moeller, H. Sasabe, J.A. Wilder, Structural Relaxation in Vitreous Materials, Annals of the New York Academy of Sciences, 279 (1976) 15-35.

[14] M. Yun, N. Jung, C. Yim, S. Jeon, Nanomechanical thermal analysis of the effects of physical aging on glass transitions in PS/PMMA blend and PS-PMMA diblock copolymers, Polymer, 52 (2011) 4136-4140.

[15] G.M. Gusler, G.B. McKenna, The craze initiation response of a polystyrene and a styrene-acrylonitrile copolymer during physical aging, Polymer Engineering and Science, 37 (1997) 1442-1448.

[16] H. Alata, T. Aoyama, Y. Inoue, Effect of Aging on the Mechanical Properties of Poly(3-hydroxybutyrate-co-3hydroxyhexanoate), Macromolecules, 40 (2007) 45464551.

[17] K. Engelsing, G. Mennig, Influence of the injection molding process on the free volume and its effect on the creep behavior of styrene-acrylonitrile copolymer, Mechanics aof Time-Dependent Materials, 5 (2001) 27-38.

[18] C.G. Robertson, G.L. Wilkes, Physical aging behavior of miscible blends of poly(methyl methacrylate) and poly(styrene-co-acrylonitrile), Polymer, 42 (2001) 15811589.

[19] J. Vernel, R.W. Rychwalski, V. Pelisek, P. Saha, M. Schmidt, F.H.J. Maurer, Physical aging in poly(ethylene oxide) atactic-poly(methyl methacrylate) blends, Thermochinmica Acta, 342 (1999) 115-137.

[20] C. Tsitsilianis, P. Papaioannou, Physical aging in block copolymers by thermal analysis, International Journal of Polymer Analysis and Characterization, 1 (1995) 63-73. [21] V. Gianotti, D. Antonioli, K. Sparnacci, M. Laus, T.J. Giammaria, F.F. Lupi, G. Seguini, M. Perego, On the Thermal Stability of PS-b-PMMA Block and P(S-r-MMA) Random Copolymers for Nanopatterning Applications, Macromolecules, 46 (2013) 8224-8234.

[22] D. Cangialosi, A. Alegría, J. Colmenero, Effect of nanostructure on the thermal glass transition and physical aging in polymer materials, Progress in Polymer Science, 54-55 (2016) 128-147.

[23] Y. Guo, R.D. Priestley, Structural relaxation of confined glassy polymers, in: S. Napolitano (Ed.) Nonequilibrium phenomena in confined soft matter, Springer, Switzerland, 2015, pp. 47-88.

[24] D. Cangialosi, V.M. Boucher, A. Alegría, J. Colmenero, Enhanced physical aging of polymer nanocomposites: the key role of the area to volume ratio, Polymer, 53 (2012) 1362-1372.

[25] V.M. Boucher, D. Cangialosi, A. Alegría, J. Colmenero, J. Gonzalez-Irun, L.M. Liz-Marzan, Accelerated physical aging in PMMA/silica nanocomposites, Soft Matter, 6 (2010) 3306-3317.

[26] V.M. Boucher, D. Cangialosi, A. Alegría, J. Colmenero, I. Pastoriza-Santos, L.M. Liz-Marzan, Physical aging of polystyrene/gold nanocomposites and its relation to the calorimetric Tg depression, Soft Matter, 7 (2011) 3607-3620. 